\title{
Effect of Mobile Phone Text Messages Reminders on Uptake of Routine Immunization among Children in Pakistan-Study Protocol for a Randomized Clinical Trial
}

\author{
Kazi AM*, Murtaza A, Kazi AN, Khurram Z, Hussain K and Ali SA
}

Division of Maternal and Child Health, Department of Paediatrics and Child Health, The Aga Khan University, Karachi, Pakistan

\begin{abstract}
Introduction: Routine Immunization (RI) is considered one of the best public health interventions to decrease child morbidity and mortality. The RI coverage in Pakistan is still well below the desired level, leading to continued polio transmission, large measles outbreaks and thousands of deaths from vaccine-preventable illnesses. Different innovative and cost effective strategies are required to look into for enhancement in vaccination uptake and coverage. We plan to test the effectiveness of reminders to parents/caregivers on cell phones through short messaging system (SMS) in improving vaccine uptake and on-time routine immunization for children in Pakistan.
\end{abstract}

Methods and Analysis: A parallel-randomized controlled trial will be conducted to assess the effectiveness of short messaging service (SMS) text reminders on the mobile phones in improving the uptake and on-time vaccination for routine immunization in children at 6,10 and 14 weeks of age according to the EPI schedule in Pakistan. The trial consists of 300 children with allocation of parallel 1:1 to the SMS reminders or to standard care (control group). The intervention arm in addition to the standard counseling will receive four SMS reminders according to the language preference in the week that the enrolled child is due for routine immunization at 6,10 and 14-week schedule. The primary outcome is to evaluate whether text reminders can improve uptake and on-time vaccination for routine immunization at 6,10 and 14 weeks' schedule. Assumptions used for calculating sample size are increase in coverage rate from $60 \%$ to $80 \%$, power at 0.8 , alpha error at 0.05 and allowing for $10 \%$ dropout.

Ethics and Dissemination: Study protocol and associated study instruments, will be submitted to the Aga Khan University's Ethical Review Committee for approval before commencement of any study activities. Results will be disseminated to pediatric and public health communities in Pakistan through scientific meetings.

\section{Trial Registration}

Clinical Trial. Gov, registration number is NCT018595

\section{Strengths}

- We plan to test the effectiveness of Short Message System (SMS) reminders to parents/caregivers on cell phones in improving the uptake and on-time RI for children in Pakistan. SMS text reminders for RI can potentially be used in connecting health care services to parents and caregivers of children eligible for RI directly detouring different hurdles confronted during physical visits $[1,2]$.

- In places where maternal and child services are available and affordable but not availed, mHealth can have a significant role in improving the coverage [3]. In this study we evaluated the role of SMS texts reminders for improvement in RI coverage at 6,10 and 14 weeks of life.

- With increased shared phone access and acceptance of technological advances there is a higher chance of improving the reach to majority of population. This provides great opportunity to improve engagement through text reminders for completion of RI of child after birth.

- Pakistan being the major contributor for the polio epidemic, any strategy to support and improvement in the RI will have a direct impact on the Polio end game strategy.

\section{Limitations}

A small sample size; the sample size estimation was done with assumption of $20 \%$ increase in coverage of the intervention arm. Participants will be recruited from the catchment population of the health demographic surveillance area, of a peri-urban population.

\section{Introduction}

Vaccines are one of the most successful and cost effective public health interventions that have immensely reduced global child morbidity and mortality [4]. The World Health Organization (WHO) initiated the expanded program of immunization (EPI) for routine immunization (RI) in 1974 in an attempt to vaccinate and protect as many children as possible [5]. RI among children according to EPI schedule is free of cost [6]. Despite this an estimated 1.5 million children globally still die due to vaccine preventable diseases each year [7]. In addition, the global measles outbreaks, continuation of polio epidemic and high vaccination drop out further increases the burden of vaccine preventable diseases in children [8]. WHO as part of global effort to increase global vaccination coverage has launched global vaccine action plan (GVAP), which recommends a target of $90 \%$ for national vaccination coverage and at least $80 \%$ for district level by 2020 [9]. Hence, different innovative and cost effective strategies are required to look into for enhancement in vaccination uptake and coverage.

*Corresponding author: Kazi AM, Division of Maternal and Child Health, Department of Paediatrics and Child Health, The Aga Khan University, Stadium Road, PO box 3500, Karachi, 74800, Pakistan, Tel: 9234862343; Fax: 92 34934294; E-mail: Momin.kazi@aku.edu

Received June 13, 2016; Accepted July 23, 2016; Published July 28, 2016

Citation: Kazi AM, Murtaza A, Kazi AN, Khurram Z, Hussain K, et al. (2016) Effect of Mobile Phone Text Messages Reminders on Uptake of Routine Immunization among Children in Pakistan-Study Protocol for a Randomized Clinical Trial. J Vaccines Vaccin 7: 333. doi: 10.4172/2157-7560.1000333

Copyright: $\odot 2016$ Kazi AM, et al. This is an open-access article distributed under the terms of the Creative Commons Attribution License, which permits unrestricted use, distribution, and reproduction in any medium, provided the original author and source are credited. 
Citation: Kazi AM, Murtaza A, Kazi AN, Khurram Z, Hussain K, et al. (2016) Effect of Mobile Phone Text Messages Reminders on Uptake of Routine Immunization among Children in Pakistan-Study Protocol for a Randomized Clinical Trial. J Vaccines Vaccin 7: 333. doi: 10.4172/21577560.1000333

Page 2 of 7

Pakistan ranks $4^{\text {th }}$ in child mortality worldwide, with over $60 \%$ of all deaths due to infectious diseases: many of which are vaccinepreventable. The immunization coverage in Pakistan is estimated to be $59 \%$, which is still well below the desired level, leading to continued polio transmission, large measles outbreaks and thousands of deaths from vaccine-preventable illnesses [10]. In addition, Pakistan is the major Polio epidemic country and the only place requiring proof of polio vaccination for international travel [11]. Improved RI coverage is recommended as the priority public health strategy, to reduce vaccine preventable diseases and eradicate polio in Pakistan and worldwide.

There has been a dramatic increase in the mobile phone usage with around 7 billion mobile phone subscribers globally, with major contribution from users in developing countries comprising of more than $80 \%$ of the 660 million new subscriptions in $2011[12,13]$. It is reported that by January 2014 there is $93 \%$ penetration of mobile phones worldwide [14,15]. Around 140 million mobile phone subscribers are currently enrolled in Pakistan: a drastic influx of mobile phone use similar to other Low Middle Income Countries (LMICs) with mobile phone penetration density being $76 \%$ in 2013 . There is also surge in use of short messaging service (SMS), with 237.58 billion person-to-person SMS generated in 2011 estimating to around 175 SMS per mobile phone on a monthly basis in Pakistan [16]. Importantly, the use of cell phones has increased in all socio-economical strata [17].

SMS text messages can be extremely effective for mobile health or mHealth and have considerable impact on disease prevention efforts in developing countries [18]. SMS messages have been quite successful to remind patients for medical appointments, clinical care intervention to increase adherence to treatment programs, and monitoring and promoting preventive health behaviors [19-21]. Mobile phone and SMS texting provides excellent solution to challenges like travel and complex intercultural contact [18].

Availability on all mobile phones, including cheap low-end handsets, makes SMS to be sent in near-real time to thousands of people with tailored messages according to user's requirement. Even if the phone is switched off or silent, message can be read and replied at one's convenience. The cost of SMS is less than the phone call and the message can be conveyed with minimum intrusiveness. A normal SMS costs less than one USD cent to send (receiving is free) and rates are much cheaper for bulk SMS packages in Pakistan. Due to the element of portability mobile phone reaches a larger population than the Internet, and thereby often-reducing time, distance and cost to access [18].

We previously conducted a study on monitoring the polio supplementary immunization activities using an automated SMS system in Karachi, Pakistan [22]. The study concluded that a two-way SMS-based system has the advantages of low cost, fair representative sampling of the population, low burdens on monitoring staff and low risk of the coverage being exaggerated. These advantages make SMS based monitoring system an attractive alternative to more conventional methods of SIA monitoring, in Pakistan and elsewhere. This model has been quite successful, and similarly we would like to evaluate whether SMS reminders increase RI coverage rates in children. We plan to test the effectiveness of reminders to parents/caregivers on cell phones through SMS reminders in improving vaccine uptake and on-time RI for children in Pakistan.

\section{Methods}

\section{Research hypothesis}

To prove that the RI uptake and compliance according to the schedule can be increased through SMS reminders in LMICs and to incorporate this program within the existing health systems by collecting mobile phone data of parents and caregivers of children less than 6 weeks of age.

\section{Primary objectives}

To assess the effectiveness of SMS reminders on mobile phones in improving the uptake for RI in children at 6,10 and 14 weeks of age according to the EPI schedule in Pakistan

\section{Secondary objectives}

To assess the effectiveness of SMS reminders on mobile phones in improving the on-time visit for RI in children at 6,10 and 14 weeks of age

To develop a low cost automated SMS text messaging computerized program with simple understandable messages according to language preferences

\section{Study design}

It is a randomized clinical trial and our experimental plan has three components:

A baseline survey of parents/caregivers to explore preferences related to SMS reminders for immunization.

A computerized application will be developed for SMS reminders scheduling. This application will contain the phone numbers, SMS text message information, language preferences and date on which the message will be sent. It will be interfaced with the gateway to send the message.

Parents/caregivers of children randomized in the intervention arm will be sent SMS reminders of RI visits at 6,10 and 14 weeks of life (Table 1).

\section{Study site}

This study will be carried out in an urban- squatter settlement area of Ibrahim Haidry (IH) union council (UC) in Karachi. This is part of Aga Khan University's Department of Pediatrics and Child Health's health demographic surveillance (HDSS) on maternal and child health being conducted since 2008 . IH is a low- income community with total population of the active surveillance catchment area is 120,725. About 2,000 pregnant women and 1900 newborns are added to the surveillance system annually.

\section{Study population}

Infants as part of the HDSS cohort conducted by the Department of Pediatrics in IH catchment area will be recruited for this study.

\section{Sample size calculations}

Target recruitment would be 300 infants per site; 150 in each arm of the study. Assumptions used for calculating sample size are increase in coverage rate from $60 \%$ to $80 \%$, power at 0.8 , alpha error at 0.05 and allowing for $10 \%$ dropout.

\section{Enrollment criteria}

Inclusion criteria: The inclusion criteria include a child being less than 14 days of age with parent/guardian or at least one person in the household having a working mobile phone connection and parent/ guardian providing consent to participate in the study. 
Citation: Kazi AM, Murtaza A, Kazi AN, Khurram Z, Hussain K, et al. (2016) Effect of Mobile Phone Text Messages Reminders on Uptake of Routine Immunization among Children in Pakistan-Study Protocol for a Randomized Clinical Trial. J Vaccines Vaccin 7: 333. doi: 10.4172/21577560.1000333

Page 3 of 7

\begin{tabular}{|c|c|c|c|c|}
\hline DISEASE & CAUSE OF INFECTION & VACCINE & DOSES & AGE OF ADMINISTRATION \\
\hline Childhood TB & Bacteria & BCG & 1 & Soon after birth \\
\hline Poliomyelitis & Virus & OPV & 4 & $\begin{array}{l}\text { OPV0: soon after birth } \\
\text { OPV1: } 6 \text { weeks } \\
\text { OPV2: } 10 \text { weeks } \\
\text { OPV3: } 14 \text { weeks }\end{array}$ \\
\hline Diphtheria & Bacteria & \multirow{5}{*}{$\begin{array}{l}\text { Pentavalent vaccine } \\
(\mathrm{DTP}+\mathrm{Hep} \mathrm{B}+\mathrm{HiB})\end{array}$} & \multirow{5}{*}{3} & \multirow{5}{*}{$\begin{array}{l}\text { Pental 1: } 6 \text { weeks } \\
\text { Pental 2: } 10 \text { weeks } \\
\text { Pental 3: } 14 \text { weeks }\end{array}$} \\
\hline Tetanus & Bacteria & & & \\
\hline Pertussis & Bacteria & & & \\
\hline Hepatitis B & Virus & & & \\
\hline Hib pneumonia and meningitis & Bacteria & & & \\
\hline Measles & Virus & Measles & 2 & $\begin{array}{l}\text { Measles 1: } 9 \text { months } \\
\text { Measles 2: } 15 \text { months }\end{array}$ \\
\hline
\end{tabular}

* Pneumococcal vaccine PCV10 was not added at the time of the study.

Table1: Childhood Immunization Schedule for Pakistan.

Exclusion criteria: The exclusion criteria include a child being more than 14 days of age with no valid mobile phone connection in the house and parent/guardian not providing consent to participate in the study.

\section{Sampling method/technique}

List of infants from the IH study catchment area will be shared on daily by HDSS team. The study staff will visit the homes of infants and offer participation to the parents in the study. After informed consent, the mobile phone data of parent/caretaker will be recorded along with address.

\section{Recruitment process}

The study staff will approach the families who have an infant less than 2 weeks of life. Parents or caretaker will be explained about the study objectives. If the parent/caretaker is interested in the study, infant will be evaluated for enrollment. After meeting the eligibility criteria, informed consent will be obtained and the infant will be enrolled in the study. Eligible infants will be randomly allocated to one of the two groups using randomly generated computer assignments. Baseline information will be collected on socio-demographics background, mobile phone related information, SMS text message language preferences and household's global positioning system (GPS) coordinates. Once the target of 300 infants is reached, the enrollment process will be stopped.

\section{Randomization and allocation}

This randomized trial will consist of 300 infants and include two intervention arms with allocation of parallel 1:1 to the SMS reminder or to standard care (control group). After informed consent eligible infants will be randomly allocated into two groups using randomly generated computer assignments, allocated in sealed opaque envelopes to be opened at the time of enrolment. IDs will be generated in blocks with 6 cases in each block. (Figure 1)

\section{Intervention}

An initial baseline survey of the infant household will be conducted which will include information on basic demographics and mobile phone related information e.g. distribution of gender, education level, preferred language for SMS text, relationship of the person with child whose mobile phone number is provided, time preferred for receiving SMS text and household's GPS coordinates on a smart phone (Figures 2, Table 2).

Control group: The control group will receive one-time standard verbal counseling at the time of initial visit and timing for EPI vaccines at 6,10 and 14 weeks as recommended by EPI government of Pakistan.
Intervention group: The intervention arm will, in addition to the standard counseling, receive four SMS reminders according to the language preference, in the week that the enrolled child is due for the EPI vaccines according to the EPI schedule. The content of the text message will be "“Child name" is due for 6-week vaccination, immediately take your child to the nearest EPI center". Same message will be sent for 10 and 14-week schedule, with timing of the week changed accordingly (Figures 3). A second interview will be conducted at 18 weeks of age of enrolled child, the study team will again visit each study subject and document information regarding EPI RI scheduled at 6,10 and 14 weeks in both intervention and control arm. Since a gap of 4 weeks is given between each vaccination dose we want to give same amount of time for capturing data after the 14-week vaccination schedule. Vaccination records will be verified by physically looking at the EPI immunization cards of the children and record in the questionnaire.

\section{Outcomes}

- The primary outcome is to evaluate whether SMS reminders can improve uptake of RI at 6,10 and 14 weeks of age according to the EPI

- The secondary outcome is to evaluate whether SMS reminders can improve on-time visits for RI in children at 6,10 and 14 weeks of age

\section{Data Entry, Cleaning and Management}

Baseline and end line data will be collected on a smart phone device. The entry program is designed to capture data as well as the location of the interviewer along with some monitoring parameters. Each child will be given a structured unique identifier. One child per household will be selected. In a household, where there was more than one (less than 14 days of life), a random selection will made by a program designed in the mobile phone device. Business rules, skips and consistency checks will be incorporated and important fields will be marked as must enter in the questionnaire to maintain data collection quality. Visual basic application script will be added in the backend to support such complex functionalities. The database will reside on a central computer managed by the study staff (Figure 4).

\section{Automated Gateway based SMS text messaging reporting system}

An automated SMS system will be designed using the secure web portal, which will be accessible through the password protected website. A program will be designed to generate a list of participants to whom the messages will be sent according to the EPI schedule and their 
Citation: Kazi AM, Murtaza A, Kazi AN, Khurram Z, Hussain K, et al. (2016) Effect of Mobile Phone Text Messages Reminders on Uptake of Routine Immunization among Children in Pakistan-Study Protocol for a Randomized Clinical Trial. J Vaccines Vaccin 7: 333. doi: 10.4172/21577560.1000333

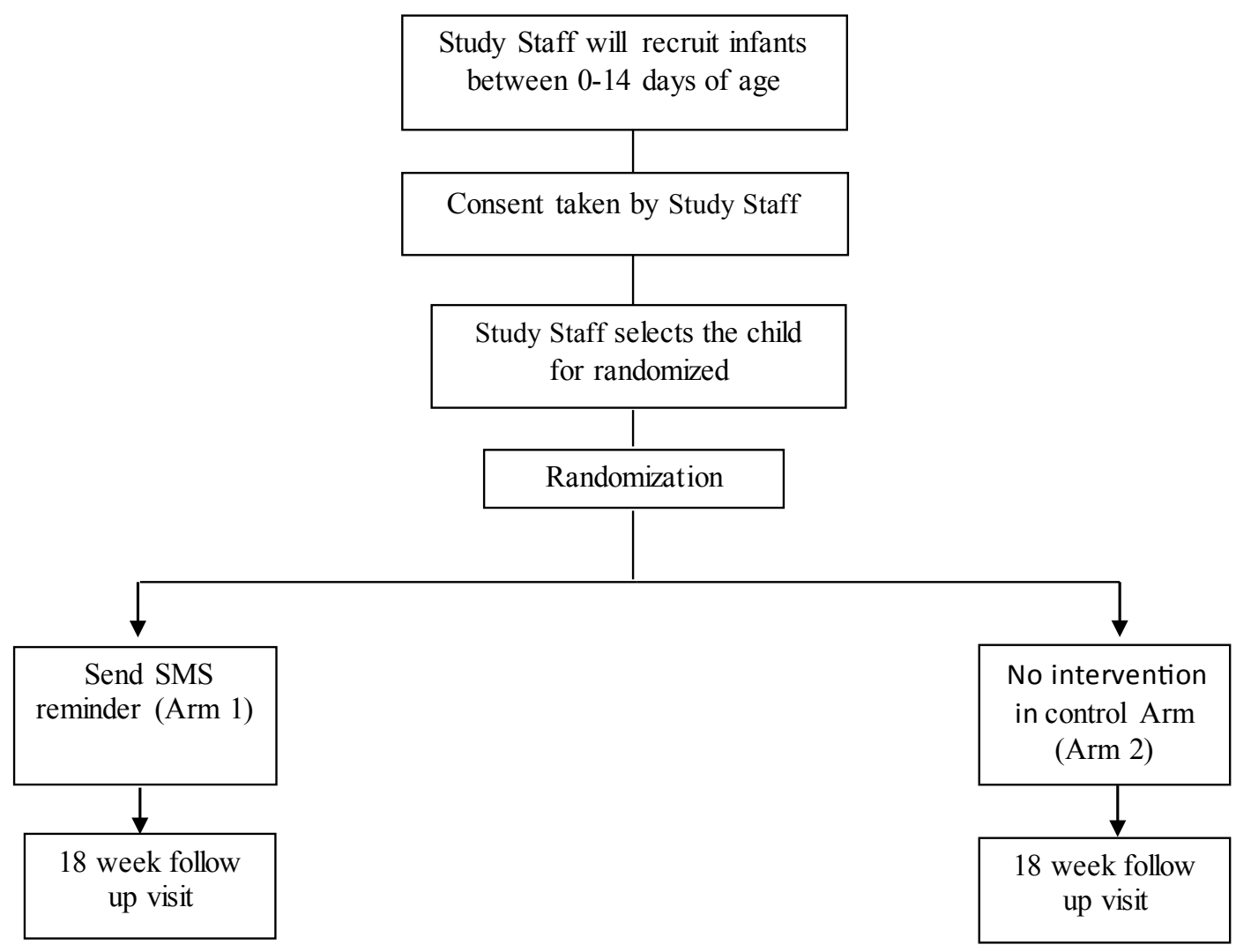

Figure 1: Randomization of Infants in the Two Arms.

\begin{tabular}{|c|c|c|c|c|}
\hline Treatment Arm & Number of participant & $\begin{array}{c}\mathbf{6} \text { Week } \\
(\mathbf{4 1 , 4 2 , 4 3 , 4 4 )}\end{array}$ & $\begin{array}{c}\mathbf{1 0} \text { Week } \\
\mathbf{( 6 9 , 7 0 , 7 1 , 7 2 )}\end{array}$ & $\begin{array}{c}\mathbf{1 4} \text { Week Week } \\
\mathbf{( 9 7 , 9 8 , 9 9 , 1 0 0 )}\end{array}$ \\
\hline $\begin{array}{c}\text { Arm 1 } \\
\text { (Intervention) }\end{array}$ & 150 & Standard EPI reminder SMS & Standard EPI reminder SMS & Standard EPI reminder SMS \\
\hline $\begin{array}{c}\text { Arm 2 } \\
\text { (Control) }\end{array}$ & 150 & One time counseling at the baseline survey & Follow up visit \\
\hline
\end{tabular}

*Standard EPI in Pakistan is OPV plus BCG at birth; DPT-Hep-B-Hib and OPV at 6, 10, and 14 weeks, and measles at 9 months and second year of life Table 2: Enrollment Schedule.

language preference of the SMS text. A list will be uploaded to the SMS gateway on daily basis.

A SMS gateway will be used to send SMS text messages unlike the conventional system i.e. sending SMS text messages via mobile phones or SMS modem attached to the computer generating the scheduled SMS. In the conventional method the recipient receives message from a normal mobile phone number, which if not saved in mobile will appear as a unknown number and the SMS text message delivery is not reliable. This usually happens in case of bulk SMS, when multiple users are sent SMS through mobile phone or SMS modem. The other down size to this is that the mobile phone operator drops such messages considering them as spam text messages, and the recipient does not receive SMS. Considering these limitations, a mobile gateway which is tough expensive but much reliable and faster in message delivery will be used to send the SMS text messages. SMS text messages sent through gateway can be tagged with a short name like 'AKU', 'Reminder' etc. so that the user is more comfortable and considers it from a reliable and trust worthy source.

A reporting module will also be developed to monitor the data captured through the cell phones based questionnaires for baseline and follow up interviews. This system will report details regarding SMS messages sent to the participants on daily basis e.g. details on SMS sent at 6,10 and 14 weeks, language sent in, children remaining etc. This web-based system will support to keep a track on the project ongoing activities and systems in real time. The web link will be password protected and will be accessed through web portal only to the study related personnel.

\section{Analysis}

Data analysis will be done using Statistical Package for Social Sciences for Windows version 19 (IBM ${ }^{\circ}$ SPSS ${ }^{\circ}$ Statistics, IBM Corporation, Somers, NY, USA). Characteristics regarding sociodemographics, mobile phone use and immunization status will be expressed in percentages across the intervention and control arms. For assessing comparability of the data on socio-demographics and mobile phone use across the 2 arms and vaccination status on the defined time intervals $(6,10$ and 14 weeks after birth), chi-square tests of independence will be employed. P-value, if found less than 0.05 , will be considered significant. 


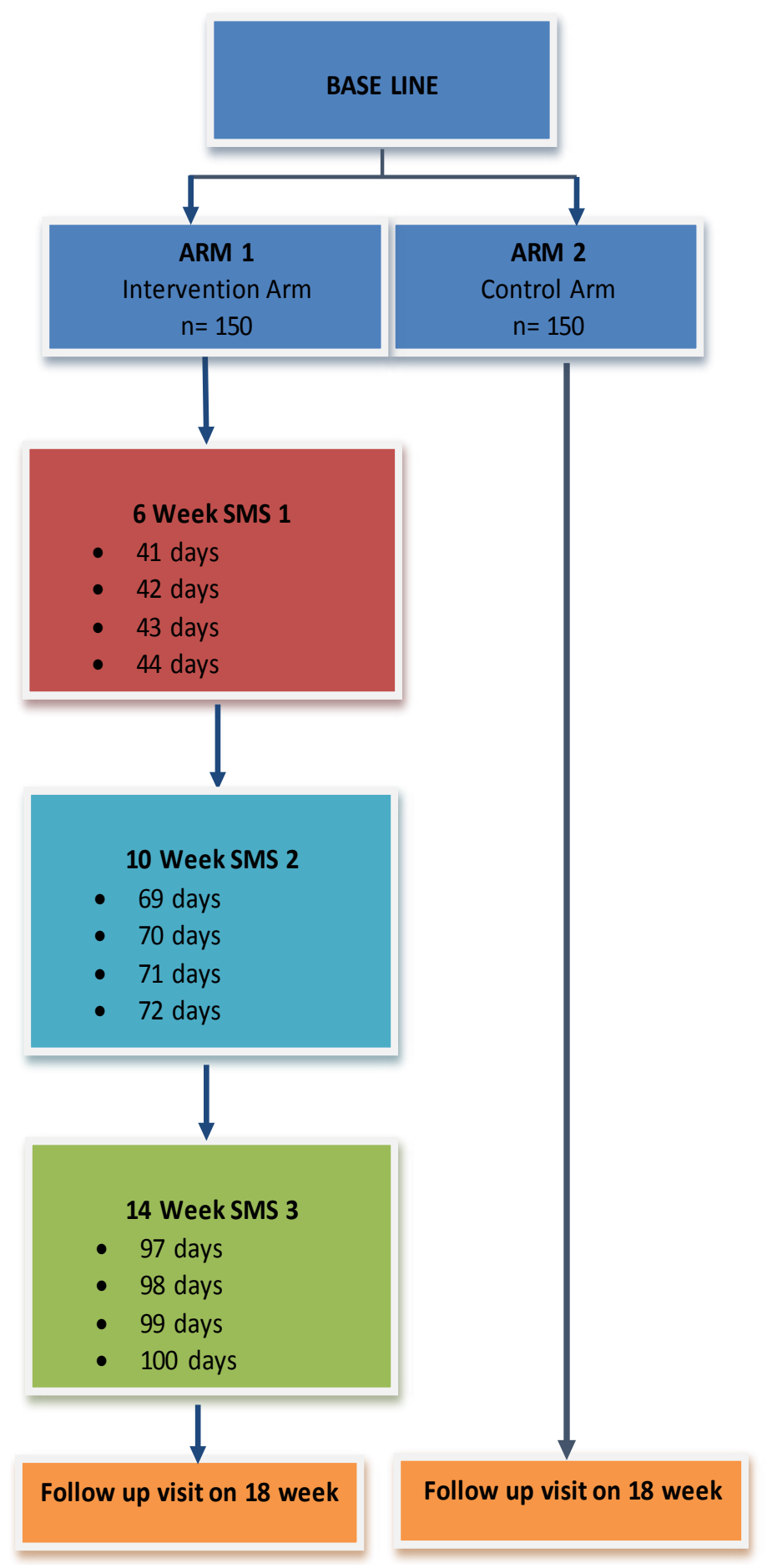

Figure 2: SMS reminder schedule in study.

\section{Ethics and Dissemination}

Institutional review board: Study protocol and associated study instruments, including consent forms in English and local language will be submitted to the Aga Khan University's Ethical Review Committee for approval before commencement of any study activities. Same documents will be submitted to World Health Organization ethical review committee for approval. The study team, including paramedics and community health workers will be trained in human subject protection.

\section{Patient data confidentiality}

All the research documents will be held confidential and only shared with individuals who are directly involved in the study. Study questionnaire data in the mobile phones will be transferred 
Citation: Kazi AM, Murtaza A, Kazi AN, Khurram Z, Hussain K, et al. (2016) Effect of Mobile Phone Text Messages Reminders on Uptake of Routine Immunization among Children in Pakistan-Study Protocol for a Randomized Clinical Trial. J Vaccines Vaccin 7: 333. doi: 10.4172/21577560.1000333

\begin{tabular}{|c|c|c|c|}
\hline Roman Urdu (Length=1) & English & Urdu(Length $=2$ ) & Sindhi(Length=2) \\
\hline $\begin{array}{l}6 \text { Week SMS : - } \\
\text { Apnay Bachay ko } 6 \text { haftay ki umar } \\
\text { walay zaruri hifazati tekay } \\
\text { lagwanay kay liye forun karebi } \\
\text { hifazati tekun kay markaz } \\
\text { ley jayen. }\end{array}$ & $\begin{array}{l}6 \text { Week SMS :- } \\
\text { Your baby is due for } \\
6 \text { week vaccination } \\
\text { immediately take your } \\
\text { child to the nearest } \\
\text { EPI center. }\end{array}$ & 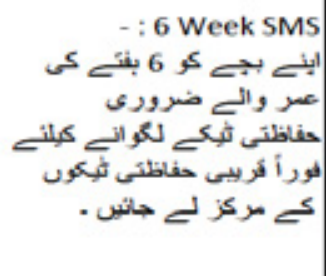 & 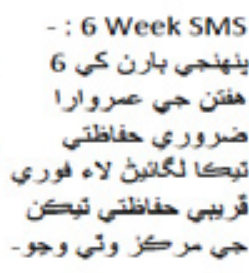 \\
\hline $\begin{array}{l}\text { 10Week SMS :- } \\
\text { Apnay Bachay ko } 10 \text { haftay ki umar } \\
\text { walay zaruri hifazati tekay } \\
\text { lagwanay kay liye forun karebi } \\
\text { hifazati tekun kay markaz } \\
\text { ley jayen. }\end{array}$ & $\begin{array}{l}10 \text { Week SMS :- } \\
\text { Your baby is due for } \\
10 \text { week vaccination } \\
\text { immediately take your } \\
\text { child to the nearest } \\
\text { EPI center. }\end{array}$ & 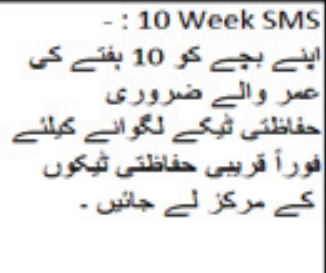 & 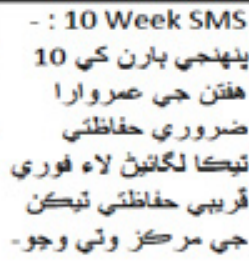 \\
\hline $\begin{array}{l}14 \text { Week SMS :- } \\
\text { Apnay Bachay ko } 14 \text { haftay ki umar } \\
\text { walay zaruri hifazati tekay } \\
\text { lagwanay kay liye forun karebi } \\
\text { hifazati tekun kay markaz } \\
\text { ley jayen. }\end{array}$ & $\begin{array}{l}14 \text { Week SMS :- } \\
\text { Your baby is due for } \\
14 \text { week vaccination } \\
\text { immediately take your } \\
\text { child to the nearest } \\
\text { EPI center. }\end{array}$ & 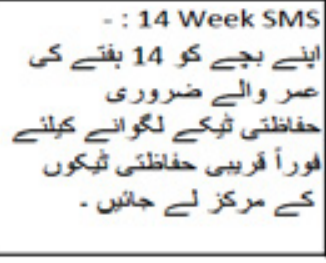 & 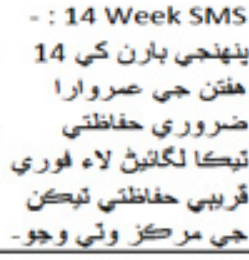 \\
\hline
\end{tabular}

Figure 3: SMS examples.

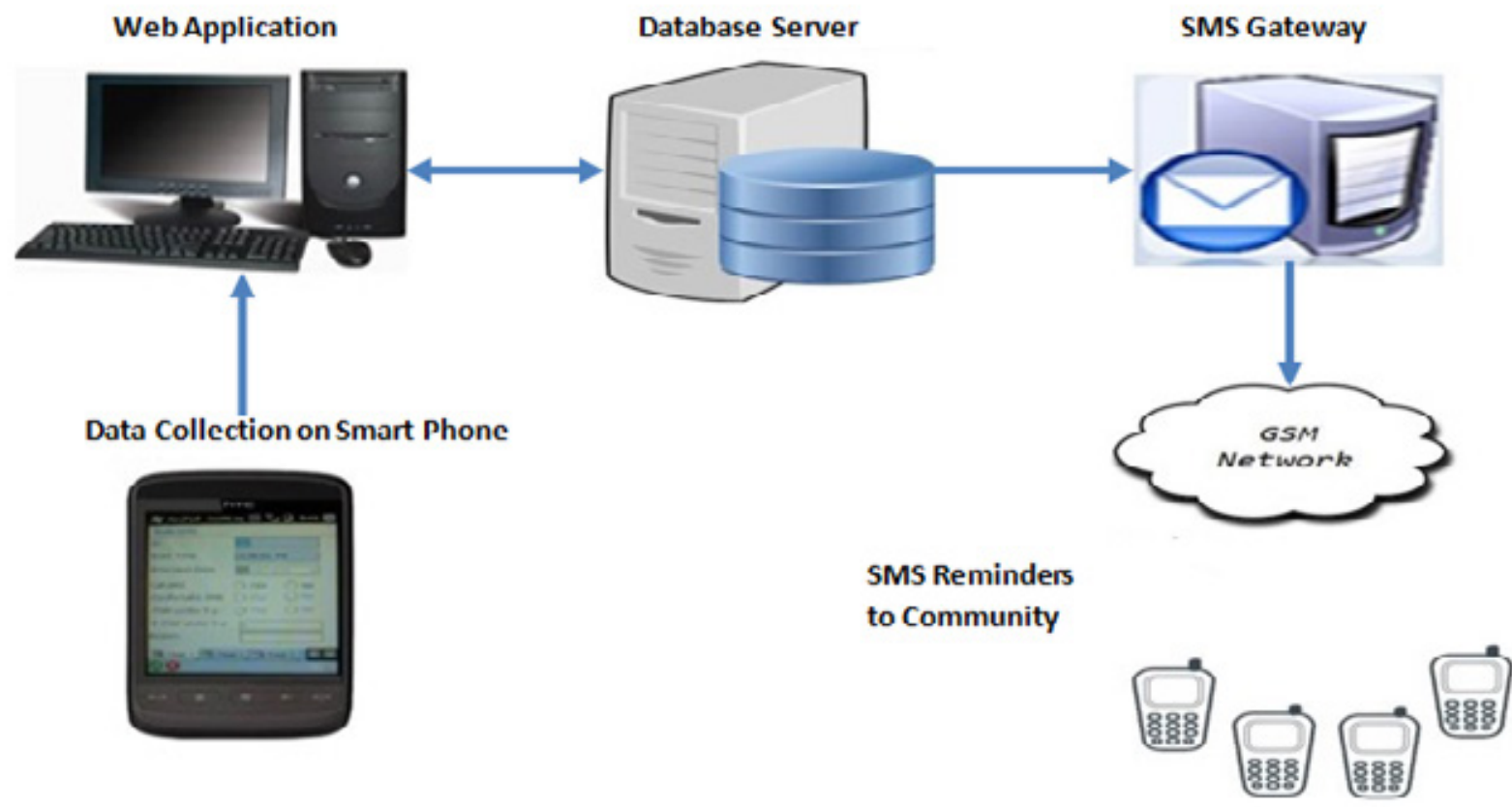

Figure 4: Data collection and Management.

on daily basis to the central database; the database will reside on a central computer managed by the study data manager. Participant's information will be given a study code and data confidentiality will be maintained at all times. No personal identifiers will be used in any reports or publications of study.

\section{Informed Consent}

Verbal explanation regarding study will be given to all the parents of the subjects and a written consent form informing them of the nature of the study, its rationale, and its risks and benefits will be signed by parents of all subjects with appropriate witness signatures. 
Citation: Kazi AM, Murtaza A, Kazi AN, Khurram Z, Hussain K, et al. (2016) Effect of Mobile Phone Text Messages Reminders on Uptake of Routine Immunization among Children in Pakistan-Study Protocol for a Randomized Clinical Trial. J Vaccines Vaccin 7: 333. doi: 10.4172/21577560.1000333

Children whose families do not wish to participate, or those who want to withdraw will continue to receive all routine care and EPI vaccines that is offered free of charge at the primary health center. There will be no negative consequences to health care services offered to these children at primary health center. Patients who dropped out during the study would be asked if there previously collected data could be used in the analysis or not.

\section{Disseminating the Result to the public}

Results will be shared with the partners at WHO and EPI Sindh. Results will also be disseminated to pediatric and public health communities in Pakistan through scientific meetings and submitted for publication in a peer-reviewed journal with an international public health audience.

\section{Study Benefits}

We will ensure that the child gets complete EPI vaccination for the entire study period. All children will undergo active surveillance and free routine health care will be provided at the department of Paediatrics Aga Khan University primary health care center within the catchment area the HDSS.

\section{Contributions}

Abdul Momin Kazi and Syed Asad Ali: study design, methodology and write up

Murtaza Ali and Abdul Nafey: methodology and design

Hussain Kalimuddin and Khurram Zubair: methodology

\section{Competing Interests}

None

\section{Acknowledgements}

None

\section{References}

1. World Health Organization (1997) Basic Newborn Resuscitation: A practical guide. Geneva.

2. Noordam AC, Kuepper BM, Stekelenburg J, Milen A (2011) Improvement of maternal health services through the use of mobile phones. Trop Med Int Health 16: 622-626.

3. Nurmatov UB, Lee SH, Nwaru BI, Mukherjee M, Grant L, Pagliari C (2014) The effectiveness of mHealth interventions for maternal, newborn and child health in low- and middle-income countries: Protocol for a systematic review and meta-analysis. Journal of Global Health 4: 010407

4. http://www.who.int/mediacentre/factsheets/fs286/en/
5. Reid M, Fleck F (2014) The immunization programme that saved millions of lives. Bull World Health Organ 92: 314-315

6. Owais A, Hanif B, Siddiqui AR, Agha A, Zaidi AK (2011) Does improving maternal knowledge of vaccines impact infant immunization rates? A community-based randomized-controlled trial in Karachi, Pakistan. BMC Public Health11: 239

7. World Health Organization (2015) Immunization coverage.

8. Anwer J, Saleem M, Babar MI, Zubair (2013) Knowledge of Vaccination in the Community: A Contributing Factor for Vaccination in Children. Pakistan Journal of Medical and Health Sciences 7.

9. Kalan R, Wiysonge CS, Ramafuthole T, Allie K, Ebrahim F, et al. (2014) Mobile phone text messaging for improving the uptake of vaccinations: a systematic review protocol. BMJ Open 4: e005130.

10. Shen AK, Fields R, McQuestion M (2014) The future of routine immunization in the developing world: challenges and opportunities. Glob Health Sci Pract 2: 381-394.

11. Kazi AM, Khalid M, Kazi AN (2014) Failure of polio eradication from Pakistan Threat to world health. J Pioneer Med Sci 4: 8-9

12. Aranda-Jan CB, Mohutsiwa-Dibe N, Loukanova S (2014) Systematic review on what works, what does not work and why of implementation of mobile health (mHealth) projects in Africa. BMC Public Health 14: 188.

13. ITU: Key statistical highlights: ITU data release June 2012 (2012) Geneva: International Telecommunication Union.

14. ITU: The World in 2013: ICT Facts and Figures (2013) Geneva: International Telecommunication Union.

15. http://www.slideshare.net/wearesocialsg/social-digital-mobile-around-theworld-january-2014.

16. http://www.pta.gov.pk/media/sms_mms_report_1303131.pdf.

17. Malik S, Chaudhry IS, Abbas Q (2009) Socio-economic Impact of Cellular Phones Growth in Pakistan: An Empirical Analysis. Pakistan Journal of Social Sciences 29: 23-37

18. Kaplan WA (2006) Can the ubiquitous power of mobile phones be used to improve health outcomes in developing countries?. Global Health 2: 9.

19. Déglise C, Suggs LS, Odermatt $P$ (2012) SMS for disease control in developing countries: a systematic review of mobile health applications. J Telemed Telecare 18: 273-281.

20. Fjeldsoe BS, Marshall AL, Miller YD (2009) Behavior change interventions delivered by mobile telephone short-message service. Am J Prev Med 36: 165173.

21. Siddiqui M, Islam MY, Mufti BA, Khan N, Farooq MS, et al. (2015) Assessing acceptability of hypertensive/diabetic patients towards mobile health based behavioral interventions in Pakistan: A pilot study. Int J Med Inform 84: 950955.

22. Kazi A, Murtaza A, Khoja S, Zaidi A, Ali S (2014) Monitoring polio supplementary immunization activities using an automated short text messaging system in Karachi, Pakistan. Bulletin of the World Health Organization 92: 220-225. 